www.nature.com $/ \mathrm{hr}$

\title{
Association of a fat-derived plasma protein omentin with carotid artery intima-media thickness in apparently healthy men
}

\author{
Rei Shibata ${ }^{1}$, Ryotaro Takahashi ${ }^{2}$, Yoshiyuki Kataoka ${ }^{1}$, Koji Ohashi ${ }^{3}$, Nobuo Ikeda ${ }^{2}$, Shinji Kihara ${ }^{4}$, \\ Toyoaki Murohara ${ }^{1}$ and Noriyuki Ouchi ${ }^{3}$
}

Obesity is causally linked with the development of atherosclerosis. Omentin is an adipocytokine whose concentrations are reduced in obese individuals. Here we examined the relationship between plasma omentin levels and carotid intima-media thickness (IMT), a marker of early atherosclerosis, in apparently healthy Japanese men. Participants were 100 Japanese men who underwent a medical checkup. Maximal IMT (max-IMT) and mean-IMT in common carotid artery were measured by highresolution carotid ultrasound system. Plasma omentin concentrations were determined by enzyme-linked immunosorbent assay. Circulating omentin levels correlated negatively with body mass index, waist circumference, fasting glucose, creatinine, max-IMT and mean-IMT, and positively with estimated glomerular filtration rates (eGFR). Single regression analysis demonstrated that max-IMT associated with age, eGFR and omentin levels, and that mean-IMT associated with age, fasting glucose, eGFR and omentin levels. Multiple regression analysis revealed that omentin levels, together with age, correlated with max-IMT and mean-IMT. Our data document that circulating omentin levels independently and negatively associate with carotid IMT in this population, suggesting that measurement of omentin may be useful for assessment of carotid IMT. Hypertension Research (2011) 34, 1309-1312; doi:10.1038/hr.2011.130; published online 4 August 2011

Keywords: adipocytokine; biomarker; carotid intima-media thickness; omentin

\section{INTRODUCTION}

Obesity, in particular, excess visceral fat depot, is highly associated with dyslipidemia, type 2 diabetes and hypertension, finally resulting in atherosclerotic cardiovascular disease. ${ }^{1}$ A growing of evidence indicates that adipose tissue affects a number of remote organs including the vasculature by producing various bioactive substances, also referred to as adipocytokines or adipokines. ${ }^{1-3}$ It has been suggested that the imbalance in the production of different adipocytokines contributes to the development of obesity-linked complications. ${ }^{1-3}$

Omentin, also known as intelectin-1, is a recently identified adipocytokine, whose expression is abundantly detected in human visceral fat tissue. ${ }^{4-6}$ Omentin is detectable in human plasma and is downregulated in subjects with obesity and type 2 diabetes. ${ }^{7,8}$ Low levels of plasma omentin are also associated with insulin resistance and endothelial dysfunction. ${ }^{9,10}$ Thus, it is conceivable that circulating omentin is associated with obesity-linked disorders. An increase in carotid arterial intima-media thickness (IMT) is an established marker of early atherosclerosis and is predictive of future cardiovascular and stroke events. ${ }^{11,12}$ In the present study, we investigated the association between circulating omentin levels and carotid IMT in apparently healthy Japanese males.

\section{METHODS}

Study population

Participants in this study were apparently healthy subjects who visited Chunichi Hospital in Nagoya for a routine checkup between 2006 and 2009. A total of 100 Japanese men with no history of cardiovascular disease, who were not taking any medication, participated in this study. All subjects enrolled in this study provided written informed consent. This study was approved by the Ethics Committee of Nagoya University School of Medicine and Chunichi Hospital.

\begin{abstract}
Measurement of carotid IMT
IMT in common carotid artery was measured by high-resolution carotid ultrasound system using the Philips iE33 system with an L11-3 probe (Philips Tokyo, Japan). IMT was defined as the distance from the leading edge of the first echogenic line to that of the second as previously described. ${ }^{12}$ The first line represents the lumen-intima interface, and the second line represents the collagen-containing upper layer of the adventitia. The carotid artery was scanned bilaterally in longitudinal and transverse planes. Maximal IMT (max-IMT) is defined as the highest IMT of several sites in bilateral carotid arteries. ${ }^{13,14}$ Mean-IMT was measured at the site of max-IMT and at two other points, $1 \mathrm{~cm}$ upstream and $1 \mathrm{~cm}$ downstream from this site, and these determinations were averaged. ${ }^{12,14}$ All measurements were performed by a
\end{abstract}

${ }^{1}$ Department of Cardiology, Nagoya University Graduate School of Medicine, Nagoya, Japan; ${ }^{2}$ Department of Cardiology, Chunichi Hospital, Nagoya, Japan; ${ }^{3}$ Department of Molecular Cardiology, Nagoya University Graduate School of Medicine, Nagoya, Japan and ${ }^{4}$ Department of Biomedical Informatics, Graduate School of Medicine, Osaka University, Osaka, Japan

Correspondence: Dr N Ouchi, Department of Molecular Cardiology, Nagoya University Graduate School of Medicine, 65 Tsurumai, Showa-Ku, Nagoya 466-8550, Japan. E-mail: nouchi@med.nagoya-u.ac.jp

Received 9 May 2011; revised and accepted 6 June 2011; published online 4 August 2011 
single expert sonographer who was blinded to the patient's clinical details. The reproducibility of the IMT was evaluated by taking two measurements 2 weeks apart on six volunteers, obtaining an intraclass correlation of 0.98 .

\section{Measurement of clinical parameters}

Venous blood samples were obtained for chemical analysis after an overnight fast. Plasma omentin levels were determined with omentin enzyme-linked immunosorbent assay kit (Bio Vendor, Candler, NC, USA). Heparin was used for plasma sampling for measurement of omentin. The intra-assay and interassay coefficients of variation of this kit were 4.1 and $4.8 \%$, respectively. Adiponectin level was determined with the use of a latex turbidometric immunoassay (Otsuka Pharmaceutical, Tokushima, Japan). Standard assays were used to measure glucose, hemoglobin Alc, insulin, total cholesterol, highdensity lipoprotein cholesterol, low-density lipoprotein cholesterol, triglycerides, creatinine and high-sensitive C-reactive protein levels. Hemoglobin Alc levels were expressed as Japan Diabetes Society values. After an appropriate rest

Table 1 Clinical parameters and their associations with omentin levels

\begin{tabular}{|c|c|c|c|}
\hline Parameters & Subjects $(n=100)$ & $r$ & P-value \\
\hline Age (years) & $52.7 \pm 8.9$ & -0.093 & 0.360 \\
\hline BMI $\left(\mathrm{kg} \mathrm{m}^{-2}\right)$ & $23.4 \pm 2.5$ & -0.248 & 0.013 \\
\hline Waist circumference $(\mathrm{cm})$ & $84.9 \pm 7.0$ & -0.200 & 0.046 \\
\hline Current smokers (\%) & 35 & & 0.654 \\
\hline Systolic BP (mm Hg) & $115.6 \pm 16.2$ & -0.058 & 0.566 \\
\hline Diastolic BP (mm Hg) & $72.4 \pm 11.4$ & -0.066 & 0.515 \\
\hline Fasting glucose $\left(\mathrm{mmol} \mathrm{I}^{-1}\right)$ & $5.60 \pm 0.3$ & -0.245 & 0.014 \\
\hline Hemoglobin A1c (\%) & $5.08 \pm 0.39$ & 0.065 & 0.519 \\
\hline Fasting insulin (mmol $\mathrm{I}^{-1}$ ) & $6.15 \pm 3.6$ & 0.093 & 0.355 \\
\hline Total cholesterol $\left(\mathrm{mmol} \mathrm{I}^{-1}\right)$ & $5.33 \pm 0.89$ & -0.089 & 0.377 \\
\hline LDL cholesterol (mmol I-1) & $3.22 \pm 0.81$ & -0.130 & 0.197 \\
\hline HDL cholesterol (mmol $\left.\mathrm{I}^{-1}\right)$ & $1.49 \pm 0.33$ & 0.059 & 0.561 \\
\hline Triglyceride $\left(\mathrm{mmol} \mathrm{I}^{-1}\right)$ & $3.24 \pm 1.81$ & -0.034 & 0.737 \\
\hline Creatinine $\left(\mu \mathrm{mol} \mathrm{I}^{-1}\right)$ & $76.0 \pm 12.4$ & -0.225 & 0.025 \\
\hline eGFR $\left(\mathrm{ml} \mathrm{min}-1\right.$ per $\left.1.73 \mathrm{~m}^{2}\right)$ & $76.2 \pm 13.3$ & 0.254 & 0.011 \\
\hline $\log \operatorname{hsCRP}\left(\mathrm{mg} \mathrm{dl}^{-1}\right)$ & $2.63 \pm 0.47$ & 0.073 & 0.470 \\
\hline Log adiponectin $\left(\mu \mathrm{g} \mathrm{ml}^{-1}\right)$ & $0.69 \pm 0.02$ & 0.099 & 0.329 \\
\hline Max-IMT (mm) & $0.74 \pm 0.16$ & -0.269 & 0.007 \\
\hline Mean-IMT (mm) & $0.66 \pm 0.13$ & -0.250 & 0.012 \\
\hline Omentin (ng ml ${ }^{-1}$ ) & $486.1 \pm 117.9$ & & \\
\hline
\end{tabular}

Abbreviations: BMI, body mass index; BP, blood pressure; eGFR, estimated glomerular filtration rates; HDL, high-density lipoprotein; hsCRP, high-sensitive C-reactive protein; LDL, low-density lipoprotein; Max-IMT, maximal intima-media thickness.

Data are presented as means + s.e.

Bold values indicate statistical significance. of $10 \mathrm{~min}$, sitting blood pressure was measured. Body mass index was calculated as the ratio of weight to squared height. Estimated glomerular filtration rates (eGFR) were calculated by the Simplified Modification of Diet in Renal Disease equation for Japanese.

\section{Statistical analysis}

Results are presented as mean \pm s.e. for continuous variables. The correlations of omentin levels with the indicated parameters were investigated by single regression analysis. Single and multiple regression analyses were performed to assess the correlations of the indicated parameters to max-IMT and mean-IMT. A value of $P<0.05$ was considered significant. All analyses were performed using JMP (version 6.03; SAS Institute, Cary, NC, USA).

\section{RESULTS}

Clinical characteristics of the study population are shown in Table 1. Mean values of body mass index, blood pressure, hemoglobin Alc, total cholesterol, low-density lipoprotein cholesterol, high-density lipoprotein cholesterol, triglyceride and creatinine were within the normal range. Max-IMT and mean-IMT were $0.74 \pm 0.16$ and $0.66 \pm 0.13 \mathrm{~mm}$, respectively. Mean plasma omentin level was $486.1 \pm 117.9 \mathrm{ng} \mathrm{ml}^{-1}$. All subjects had no plaque formation in carotid artery. Circulating omentin levels correlated negatively with body mass index, waist circumference, fasting glucose and creatinine, and positively with eGFR (Table 1). Plasma omentin levels inversely correlated with max-IMT and mean-IMT (Table 1 and Figure 1).

Results of regression analysis for max-IMT and mean-IMT are shown in Table 2. Single regression analysis for max-IMT demonstrated that age positively correlated with max-IMT and that eGFR, and omentin levels negatively correlated with max-IMT. Multiple regression analysis with age, smoking status, systolic blood pressure, fasting glucose, total cholesterol, eGFR and omentin revealed that age and omentin levels significantly associated with max-IMT. Single regression analysis for mean-IMT revealed that age and fasting glucose positively correlated with mean-IMT, and that eGFR and omentin levels inversely correlated with mean-IMT. Multiple regression analysis with age, waist circumference, smoking status, fasting glucose, eGFR and omentin revealed that age and omentin levels significantly associated with mean-IMT.

\section{DISCUSSION}

This study demonstrated that plasma levels of omentin, together with age, are independently associated with carotid IMT in apparently healthy subjects. Low levels of circulating omentin are shown to associate with type 2 diabetes. ${ }^{8}$ It has also been reported that glucose treatment reduces omentin expression and secretion in omental fat tissue explants. ${ }^{10}$ Consistent with these observations, our present data
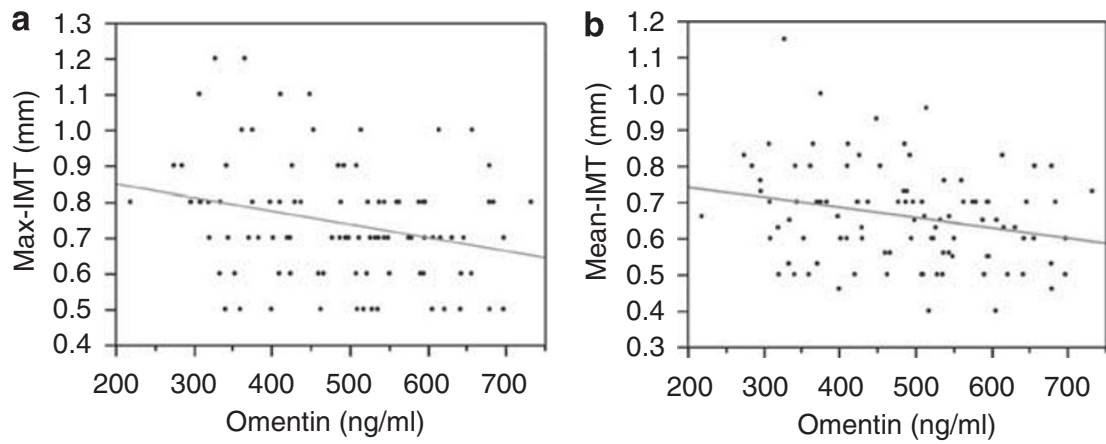

Figure 1 Correlation of plasma omentin levels with maximal intima-media thickness (max-IMT) (a) and mean-IMT (b). Plasma omentin concentrations were measured by an enzyme-linked immunosorbent assay system. A full color version of this figure is available at the Hypertension Research journal online. 


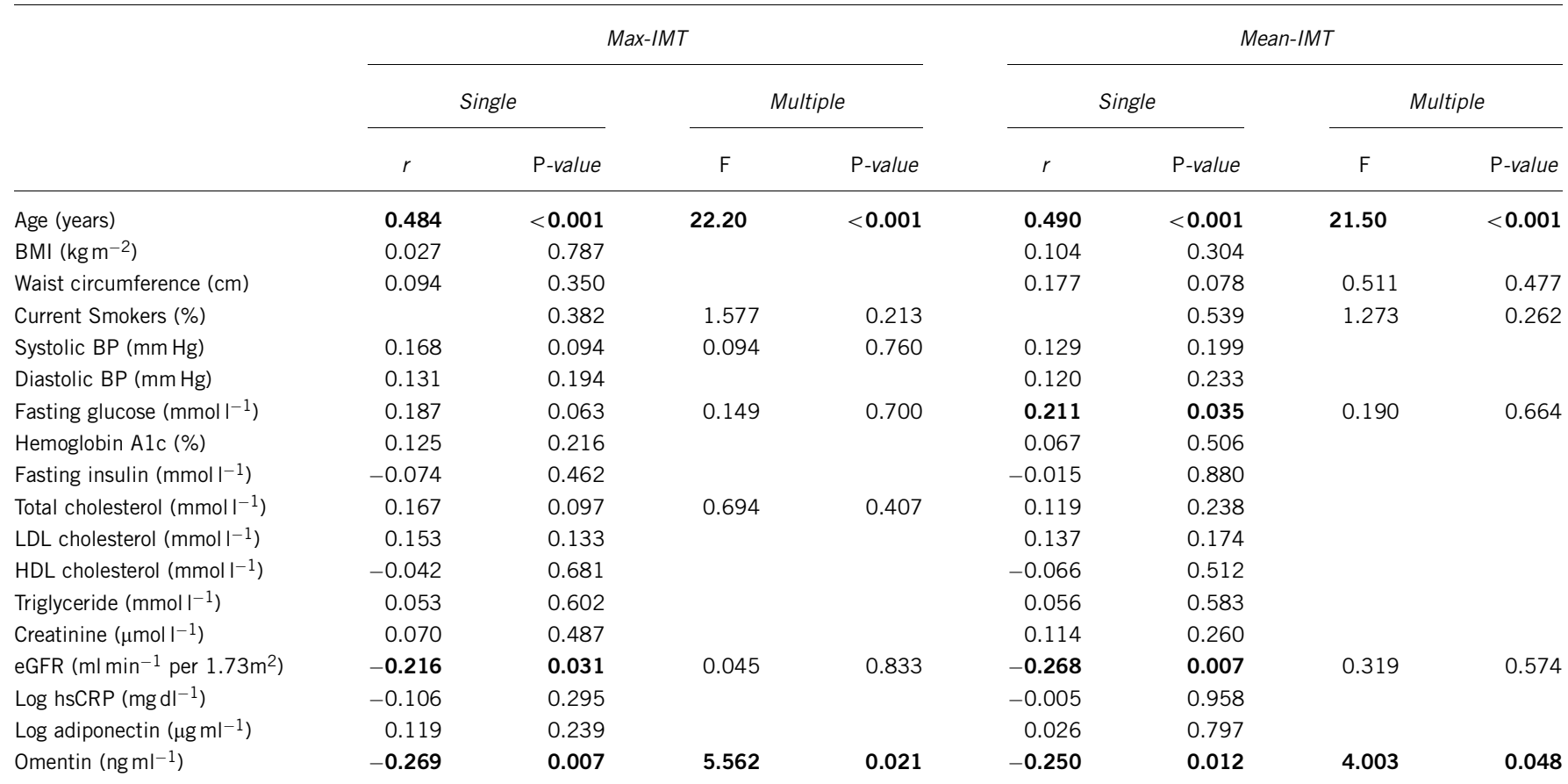

Abbreviations: BMI, body mass index; BP, blood pressure; eGFR, estimated glomerular filtration rates; HDL, high-density lipoprotein; hsCRP, high-sensitive C-reactive protein; LDL, low-density lipoprotein.

Multiple model includes smoking status and all variables at baseline with $P<0.1$ by single analysis.

Bold values indicate statistical significance.

showed that plasma omentin levels negatively associated with glucose levels. Our data also demonstrated that circulating omentin levels correlated inversely with creatinine levels, and positively with eGFR. Because glucose intolerance and renal dysfunction are shown to strongly associate with increased IMT, ${ }^{15,16}$ it is reasonable that these disease conditions could affect circulating omentin levels. However, the present study suggests that omentin levels are predictive of carotid IMT, independent of glucose levels and renal function.

A recent report showed that omentin suppresses tumor necrosis factor-stimulated cyclooxygenase- 2 expression in cultured endothelial cells through its ability to reduce c-Jun N-terminal kinase activation, ${ }^{17}$ suggesting that omentin attenuates vascular inflammation. Omentin is also shown to promote endothelial nitric oxide synthase activation in rat aorta and endothelial cells. ${ }^{17,18}$ It is well known that endothelial nitric oxide synthase has a protective role in the control of various vascular diseases including atherosclerosis. ${ }^{19}$ These findings suggest that omentin displays anti-atherogenic properties.

It has been suggested that omentin levels positively correlate with endothelium-dependent vasodilation. ${ }^{9}$ The current cross-sectional study demonstrated the negative association between plasma levels of omentin and carotid IMT. Accumulating evidence suggests that measurement of endothelial function and carotid IMT can be predictive of cardiovascular and stroke events. ${ }^{12,20,21}$ At this time, it remains unclear whether omentin acts as a predictive factor for the vascular outcomes, and this analysis requires future prospective studies.

This study has several limitations. The sample size was relatively small. This study population contained only apparently healthy males. A previous report showed that gender difference is observed in circulating omentin concentration. ${ }^{7}$ It has also been shown that carotid IMT is generally greater in men than in women. ${ }^{14}$ Although it is well established that plaque thickness is a predictor of cardiovascular risk, ${ }^{12}$ no plaque in carotid artery was observed in apparently healthy subjects in this study. Thus, future studies are needed to confirm our results in a larger unselected general population.

In conclusion, the present study indicates that circulating level of omentin is a novel indicator of carotid IMT, and that measurement of omentin levels may be valuable for evaluation of carotid IMT.

\section{CONFLICT OF INTEREST}

The authors declare no conflict of interest.

\section{ACKNOWLEDGEMENTS}

This work was supported by Grant-in-Aid for Scientific Research, and grants from Suzuken Memorial Foundation, Japan Research Foundation for Clinical Pharmacology and the Uehara Memorial Foundation to N Ouchi. R Shibata was supported with the Uehara Memorial Foundation and Japan Heart Foundation/Novartis Grant for Research Award on Molecular and Cellular Cardiology, 2011.

1 Matsuzawa Y. Therapy Insight: adipocytokines in metabolic syndrome and related cardiovascular disease. Nat Clin Pract Cardiovasc Med 2006; 3: 35-42.

2 Ouchi N, Parker JL, Lugus JJ, Walsh K. Adipokines in inflammation and metabolic disease. Nat Rev Immunol 2011; 11: 85-97.

3 Gable DR, Hurel SJ, Humphries SE. Adiponectin and its gene variants as risk factors for insulin resistance, the metabolic syndrome and cardiovascular disease. Atherosclerosis 2006; 188: 231-244.

4 Yang RZ, Lee MJ, Hu H, Pray J, Wu HB, Hansen BC, Shuldiner AR, Fried SK, McLenithan JC, Gong DW. Identification of omentin as a novel depot-specific adipokine in human adipose tissue: possible role in modulating insulin action. Am J Physiol Endocrinol Metab 2006; 290: E1253-E1261.

5 Schaffler A, Neumeier M, Herfarth H, Furst A, Scholmerich J, Buchler C. Genomic structure of human omentin, a new adipocytokine expressed in omental adipose tissue. Biochim Biophys Acta 2005; 1732: 96-102.

6 Tsuji S, Uehori J, Matsumoto M, Suzuki Y, Matsuhisa A, Toyoshima K, Seya T. Human intelectin is a novel soluble lectin that recognizes galactofuranose in carbohydrate chains of bacterial cell wall. J Biol Chem 2001; 276: 23456-23463. 
7 de Souza Batista CM, Yang RZ, Lee MJ, Glynn NM, Yu DZ, Pray J, Ndubuizu K, Patil S, Schwartz A, Kligman M, Fried SK, Gong DW, Shuldiner AR, Pollin TI, McLenithan JC. Omentin plasma levels and gene expression are decreased in obesity. Diabetes 2007; 56: 1655-1661.

8 Pan HY, Guo L, Li Q. Changes of serum omentin-1 levels in normal subjects and in patients with impaired glucose regulation and with newly diagnosed and untreated type 2 diabetes. Diabetes Res Clin Pract 2010; 88: 29-33.

9 Moreno-Navarrete JM, Ortega F, Castro A, Sabater M, Ricart W, Fernandez-Real JM. Circulating omentin as a novel biomarker of endothelial dysfunction. Obesity (Silver Spring 2011 (in press).

10 Tan BK, Adya R, Farhatullah S, Lewandowski KC, O'Hare P, Lehnert H, Randeva HS. Omentin-1, a novel adipokine, is decreased in overweight insulin-resistant women with polycystic ovary syndrome: ex vivo and in vivo regulation of omentin-1 by insulin and glucose. Diabetes 2008; 57: 801-808.

11 Chambless LE, Heiss G, Folsom AR, Rosamond W, Szklo M, Sharrett AR, Clegg LX. Association of coronary heart disease incidence with carotid arterial wall thickness and major risk factors: the Atherosclerosis Risk in Communities (ARIC) Study, 1987-1993. Am J Epidemiol 1997; 146: 483-494.

12 O'Leary DH, Polak JF, Kronmal RA, Manolio TA, Burke GL, Wolfson Jr SK. Carotid-artery intima and media thickness as a risk factor for myocardial infarction and stroke in older adults. Cardiovascular Health Study Collaborative Research Group. N Eng/ J Med 1999; 340: 14-22.

13 Baldassarre D, Amato M, Pustina L, Tremoli E, Sirtori CR, Calabresi L, Franceschini G. Increased carotid artery intima-media thickness in subjects with primary hypoalphalipoproteinemia. Arterioscler Thromb Vasc Biol 2002; 22: 317-322.
14 Yoshida S, Aihara K, Azuma H, Uemoto R, Sumitomo-Ueda Y, Yagi S, Ikeda Y, Iwase T, Nishio S, Kawano H, Miki J, Yamada H, Hirata Y, Akaike M, Sata M, Matsumoto T. Dehydroepiandrosterone sulfate is inversely associated with sex-dependent diverse carotid atherosclerosis regardless of endothelial function. Atherosclerosis 2010; 212: 310-315.

15 Wagenknecht LE, D’Agostino Jr RB, Haffner SM, Savage PJ, Rewers M. Impaired glucose tolerance, type 2 diabetes, and carotid wall thickness: the Insulin Resistance Atherosclerosis Study. Diabetes Care 1998; 21: 1812-1818.

16 Ishizaka N, Ishizaka Y, Toda E, Koike K, Seki G, Nagai R, Yamakado M. Association between chronic kidney disease and carotid intima-media thickening in individuals with hypertension and impaired glucose metabolism. Hypertens Res 2007; 30: 1035-1041.

17 Yamawaki H, Kuramoto J, Kameshima S, Usui T, Okada M, Hara Y. Omentin, a novel adipocytokine inhibits TNF-induced vascular inflammation in human endothelial cells. Biochem Biophys Res Commun 2011; 408: 339-343.

18 Yamawaki H, Tsubaki N, Mukohda M, Okada M, Hara Y. Omentin, a novel adipokine, induces vasodilation in rat isolated blood vessels. Biochem Biophys Res Commun 2010; 393: 668-672.

19 Napoli C, de Nigris F, Williams-Ignarro S, Pignalosa O, Sica V, Ignarro LJ. Nitric oxide and atherosclerosis: an update. Nitric Oxide 2006; 15: 265-279.

20 Kitamura A, Iso H, Imano H, Ohira T, Okada T, Sato S, Kiyama M, Tanigawa T, Yamagishi $\mathrm{K}$, Shimamoto T. Carotid intima-media thickness and plaque characteristics as a risk factor for stroke in Japanese elderly men. Stroke 2004; 35: 2788-2794.

21 Fathi R, Haluska B, Isbel N, Short L, Marwick TH. The relative importance of vascular structure and function in predicting cardiovascular events. J Am Coll Cardiol 2004; 43: 616-623. 